\title{
Multiple Solutions for a Class of Concave-Convex Quasilinear Elliptic Systems with Nonlinear Boundary Condition
}

\author{
Li Wang \\ School of Basic Science, East China Jiaotong University, Nanchang, China \\ Email:wangli.423@163.com
}

Received June 7, 2012; revised February 6, 2013; accepted February 13, 2013

\begin{abstract}
In this paper, a quasilinear elliptic system is investigated, which involves concave-convex nonlinearities and nonlinear boundary condition. By Nehari manifold, fibering method and analytic techniques, the existence of multiple nontrivial nonnegative solutions to this equation is verified.
\end{abstract}

Keywords: Multiple Solutions; Quasilinear Elliptic Systems; Nehari Manifold; Fibering Method

\section{Introduction}

In this article, we are interested in the existence of two nontrivial nonnegative solutions of the following problem:

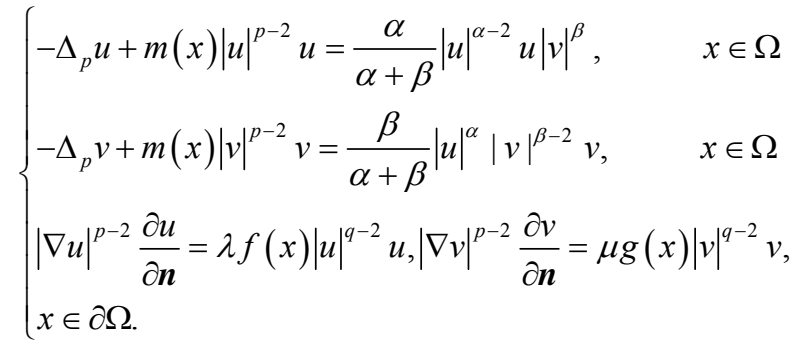

where $\Omega \subset R^{N}$ is a bounded domain with smooth boundary, $2<p<\alpha+\beta<p^{*}=\frac{N p}{N-p}$ is the critical Sobolev exponent for the embedding $W^{1, p}\left(R^{N}\right) \rightarrow L^{p}\left(R^{N}\right)$.

$1<q<p, \quad \frac{\partial}{\partial \boldsymbol{n}}$ is the outer normal derivative,

$(\lambda, \mu) \in \boldsymbol{R}^{2} \backslash\{(0,0)\}$, the weight $m(x)$ is a positive bounded function and $f(x), g(x) \in C(\Omega)$ are smooth functions which may change sign in $\Omega$. By Nehari manifold, fibering method and analytic techniques, the existence of multiple positive solutions to this equation is verified.

In recent years, there have been many papers concerned with the existence and multiplicity of positive solutions for semilinear elliptic problems. Some interesting results can be found in Garcia-Azorero et al. [1], Wu [2-4] and the references therein. More recently, Hsu [5] has considered the following elliptic system:

$$
\begin{cases}-\Delta_{p} u=\frac{2 \alpha}{\alpha+\beta}|u|^{\alpha-2} u|v|^{\beta}+\lambda|u|^{q-2} u, & x \in \Omega, \\ -\Delta_{p} v=\frac{2 \beta}{\alpha+\beta}|u|^{\alpha}|v|^{\beta-2} v+\mu|v|^{q-2} v, & x \in \Omega, \\ u=v=0, & x \in \partial \Omega .\end{cases}
$$

By variational methods, he proved that problem (1.2) has at least two positive solutions if the pair of the parameters $(\lambda, \mu)$ belongs to a certain subset of $\boldsymbol{R}^{2}$. However, as far as we know, there are few results of problem (1.1) in addition to concave-convex nonlinearities, i.e., $1<q<p$, including nonlinear boundary condition. We focus on the existence of at least two nontrivial nonnegative solutions for problems (1.1) in the present paper.

Set

$$
\begin{aligned}
\Lambda_{1} & =\left(\frac{p-q}{\alpha+\beta-q} S^{\frac{\alpha+\beta}{p}}\right)^{\frac{p}{\alpha+\beta-p}} \\
& \cdot\left(\frac{\alpha+\beta-p}{\alpha+\beta-q}\right)^{\frac{p}{p-q}} \bar{S}^{\frac{q}{p-q}}
\end{aligned}
$$

where $S, \bar{S}$ satisfy

$$
\begin{aligned}
& \left(\int_{\Omega}|u|^{\alpha+\beta} \mathrm{d} x\right)^{\frac{p}{\alpha+\beta}} S \leq \int_{\Omega}|\nabla u|^{p} \mathrm{~d} x, \\
& \left(\int_{\partial \Omega}|u|^{q} \mathrm{~d} x\right)^{\frac{p}{q}} \bar{S} \leq \int_{\partial \Omega}|\nabla u|^{p} \mathrm{~d} x,
\end{aligned}
$$

The main result of this paper is summarized in the following theorem. 
Theorem 1.1. If the parameters $\lambda, \mu$ satisfy

$$
\left(|\lambda|\|f\|_{\infty}\right)^{\frac{p}{p-q}}+\left(|\mu|\|g\|_{\infty}\right)^{\frac{p}{p-q}} \in\left(0,\left(\frac{q}{p}\right)^{\frac{p}{p-q}} \Lambda_{1}\right) \subset\left(0, \Lambda_{1}\right),
$$

then problem (1.1) has at least two solutions $(u, v)$ and $(U, V)$ satisfy $u, v, U, V \geq 0$ in $\Omega$ and $u, v, U, V \neq 0$.

It should be mentioned that the similar results about the existence of multiplicity of positive solutions for the Laplace problem with critical growth and sublinear perturbation have been discussed in the recent paper [6-8] and the reference therein.

This paper is organized as follows. Some preliminaries and properties of the Nehair manifold are established in Sections 2, and Theorems 1.1 is proved in Sections 3.

\section{Preliminaries}

Let $W^{1, p}(\Omega)$ denotes the usual Sobolev space. In the Banach space $W:=W^{1, p}(\Omega) \times W^{1, p}(\Omega)$ we introduce the norm which is equivalent to the standard one:

$$
\begin{aligned}
\|(u, v)\|_{W}^{p} & =\|u\|_{1, p}^{p}+\|v\|_{1, p}^{p} \\
& =\int_{\Omega}\left(|\nabla u|^{p}+m(x)|u|^{p}\right) \mathrm{d} x \\
& +\int_{\Omega}\left(|\nabla v|^{p}+m(x)|v|^{p}\right) \mathrm{d} x
\end{aligned}
$$

First, we give the definition of the weak solution of (1.1).

Definition 2.1. We say that $(u, v) \in W$ is a weak solution to (1.1) if for all $\left(\varphi_{1}, \varphi_{2}\right) \in W$, we have

$$
\begin{aligned}
& \int_{\Omega}\left(|\nabla u|^{p-2} \nabla u \nabla \varphi_{1}+m(x)|u|^{p-2} u \varphi_{1}\right) \mathrm{d} x \\
& =\lambda \int_{\partial \Omega} f(x)|u|^{q-2} u \varphi_{1} \mathrm{~d} s+\frac{\alpha}{\alpha+\beta} \int_{\Omega}|u|^{\alpha-2} u \varphi_{1}|v|^{\beta} \mathrm{d} x \\
& \int_{\Omega}\left(|\nabla v|^{p-2} \nabla v \nabla \varphi_{1}+m(x)|v|^{p-2} v \varphi_{2}\right) \mathrm{d} x \\
& =\mu \int_{\partial \Omega} g(x)|v|^{q-2} v \varphi_{2} \mathrm{~d} s+\frac{\beta}{\alpha+\beta} \int_{\Omega}|u|^{\alpha}|v|^{\beta-2} v \varphi_{2} \mathrm{~d} x .
\end{aligned}
$$

It is clear that problem (1.1) has a variational structure. Let $I(u, v): W \rightarrow R$ be the corresponding energy functional of problem (1.1), and it is defined by

$$
I(u, v)=\frac{1}{p}\|(u, v)\|_{W}^{p}-\frac{1}{q} R(u, v)-\frac{1}{\alpha+\beta} G(u, v),
$$

where

$$
\begin{gathered}
R(u, v)=\lambda \int_{\partial \Omega} f(x)|u|^{q} \mathrm{~d} s+\mu \int_{\partial \Omega} g(x)|v|^{q} \mathrm{~d} s, \\
G(u, v)=\int_{\Omega}|u|^{\alpha}|v|^{\beta} \mathrm{d} x .
\end{gathered}
$$

It is not difficult to verify that the functional $I$ is not bounded neither from below nor from above. So it is convenient to consider $I$ restricted to a natural constraint, the Nehari manifold, that contains all the critical points of $I$. First we introduce the following notation: for any functional $F: W \rightarrow \boldsymbol{R}$ we denote by $F^{\prime}(u, v)\left(\phi_{1}, \phi_{2}\right)$ the Gateaux derivative of $F$ at $(u, v) \in W$ in the direction of $\left(\phi_{1}, \phi_{2}\right) \in W$, and

$$
\begin{gathered}
F^{(1)}(u, v) \phi_{1}=\left.F^{\prime}\left(u+\epsilon \phi_{1}, v\right)\right|_{\epsilon=0}, \\
F^{(2)}(u, v) \phi_{2}=\left.F^{\prime}\left(u, v+\delta \phi_{2}\right)\right|_{\delta=0} .
\end{gathered}
$$

Define the Nehari manifold

$N=\left\{(u, v) \in W \backslash\{(0,0)\} \mid\left\langle I^{\prime}(u, v),(u, v)\right\rangle=0\right\}$. Note that $N$ contains all solutions of (1.1) and $(u, v) \in N$ if and only if

$$
\|(u, v)\|_{W}^{p}-R(u, v)=G(u, v) .
$$

Lemma 2.1. $I(u, v)$ is coercive and bounded below on $N$.

Proof. Suppose $(u, v) \in N$. From (2.1), the Holder inequality and the Sobolev embedding theorem, it follows that

$$
\begin{aligned}
& I(u, v) \\
= & \frac{1}{p}\|(u, v)\|_{W}^{p}-\frac{1}{q} R(u, v)-\frac{1}{\alpha+\beta} G(u, v) \\
= & \left(\frac{1}{p}-\frac{1}{\alpha+\beta}\right)\|(u, v)\|_{W}^{p}-\left(\frac{1}{q}-\frac{1}{\alpha+\beta}\right) R(u, v) \\
\geq & \frac{\alpha+\beta-p}{p(\alpha+\beta)}\|(u, v)\|_{W}^{p} \\
& -\frac{\alpha+\beta-q}{q(\alpha+\beta)} \bar{S}^{-\frac{q}{p}}\|(u, v)\|_{W}^{q}\left(|\lambda|\|f\|_{\infty}+|\mu|\|g\|_{\infty}\right) .
\end{aligned}
$$

Thus $I$ is coercive and bounded below on $N$ since $q<p<\alpha+\beta$. Define $\Phi(u, v)=\left\langle I^{\prime}(u, v),(u, v)\right\rangle$. Then for all $(u, v) \in N$ we have

$$
\begin{aligned}
& \Phi^{\prime}(u, v),(u, v) \\
& =p\|(u, v)\|_{W}^{p}-q R(u, v)-(\alpha+\beta) G(u, v) \\
& =(p-q)\|(u, v)\|_{W}^{p}-(\alpha+\beta-q) G(u, v) \\
& =[p-(\alpha+\beta)]\|(u, v)\|_{W}^{p}+(\alpha+\beta-q) R(u, v) \\
& =(p-q) R(u, v)-[p-(\alpha+\beta)] G(u, v) .
\end{aligned}
$$

Arguing as that in $[9,10]$, we split $N$ into three parts:

$$
\begin{aligned}
& N^{+}=\left\{(u, v) \in N \mid\left\langle\Phi^{\prime}(u, v),(u, v)\right\rangle>0\right\}, \\
& N^{0}=\left\{(u, v) \in N \mid\left\langle\Phi^{\prime}(u, v),(u, v)\right\rangle=0\right\}, \\
& N^{-}=\left\{(u, v) \in N \mid\left\langle\Phi^{\prime}(u, v),(u, v)\right\rangle<0\right\} .
\end{aligned}
$$

Lemma 2.2. Suppose $(u, v)$ is a local minimizer of $I$ 
on $N$ and $(u, v) \notin N^{0}$. Then $I^{\prime}(u, v)=0$ in $W^{-1}$.

Proof. If $(u, v)$ is a local minimizer for $I$ on $N$, then $(u, v)$ is a solution of the optimization problem minimize $I$ subject to

$$
\Phi(u, v)=0 .
$$

Hence, by the theory of Lagrange multipliers, there exists $\xi \in R$ such that

$$
I^{\prime}(u, v)=\xi \Phi^{\prime}(u, v) \text { in } W^{-1}(\Omega) .
$$

Here $W^{-1}(\Omega)$ is the dual space of the Sobolev space $W$. Thus,

$$
\left\langle I^{\prime}(u, v),(u, v)\right\rangle=\xi\left\langle\Phi^{\prime}(u, v),(u, v)\right\rangle
$$

But $\left\langle\Phi^{\prime}(u, v),(u, v)\right\rangle \neq 0$ since $(u, v) \notin N^{0}$. Hence $\xi=0$.

Lemma 2.3. $N^{0}=\varnothing$ for all

$$
\left(|\lambda|\|f\|_{\infty}\right)^{\frac{p}{p-q}}+\left(|\mu|\|g\|_{\infty}\right)^{\frac{p}{p-q}} \in\left(0, \Lambda_{1}\right)
$$

Proof. We argue by contradiction. Suppose that for all $\left(|\lambda|\|f\|_{\infty}\right)^{\frac{p}{p-q}}+\left(|\mu|\|g\|_{\infty}\right)^{\frac{p}{p-q}} \in\left(0, \Lambda_{1}\right)$ there is $(u, v) \in N^{0}$, then (2.3) and the Sobolev embedding theorem imply that

$$
\begin{aligned}
& (p-q)\|(u, v)\|_{W}^{p}=(\alpha+\beta-q) G(u, v) \\
& \leq(\alpha+\beta-q) S^{-\frac{\alpha+\beta}{p}}\|(u, v)\|_{W}^{\alpha+\beta},
\end{aligned}
$$

and

$$
\begin{aligned}
& (\alpha+\beta-q)\|(u, v)\|_{W}^{p} \\
& =(\alpha+\beta-q) R(u, v) \\
& \leq(\alpha+\beta-q)\left(|\lambda|\|f\|_{\infty} \int_{\partial \Omega}|u|^{q} \mathrm{~d} x+|\mu|\|g\|_{\infty} \int_{\partial \Omega}|v|^{q} \mathrm{~d} x\right) \\
& \leq(\alpha+\beta-q) \bar{S}^{\frac{-q}{p}}\left(|\lambda|\|f\|_{\infty}\|u\|_{1, p}^{q}+|\mu|\|g\|_{\infty}\|v\|_{1, p}^{q}\right) \\
& \leq(\alpha+\beta-q) \bar{S}^{\frac{-q}{p}}\left(|\lambda|\|f\|_{\infty}+|\mu|\|g\|_{\infty}\right)\|(u, v)\|_{W}^{q} .
\end{aligned}
$$

Thus from (2.4), (2.5) we have

$$
\|(u, v)\|_{W} \geq\left[\frac{p-q}{\alpha+\beta-q} S^{\frac{\alpha+\beta}{p}}\right]^{\frac{1}{\alpha+\beta-p}}
$$

and

$$
\begin{aligned}
\|(u, v)\|_{W} \leq & \left(\frac{\alpha+\beta-q}{\alpha+\beta-p}\right)^{\frac{1}{p-q}} \bar{S}^{-\frac{q}{p(p-q)}} \\
& \cdot\left[\left(|\lambda|\|f\|_{\infty}\right)^{\frac{p}{p-q}}+\left(|\mu|\|g\|_{\infty}\right)^{\frac{p}{p-q}}\right]^{\frac{1}{p}} .
\end{aligned}
$$

Consequently,

$$
\begin{aligned}
& \left(|\lambda|\|f\|_{\infty}\right)^{\frac{p}{p-q}}+\left(|\mu|\|g\|_{\infty}\right)^{\frac{p}{p-q}} \\
& \geq\left(\frac{p-q}{\alpha+\beta-q} S^{\frac{\alpha+\beta}{p}}\right)^{\frac{p}{\alpha+\beta-p}}\left(\frac{\alpha+\beta-p}{\alpha+\beta-q}\right)^{\frac{p}{p-q}} \bar{S}^{\frac{q}{p-q}} \\
& =\Lambda_{1},
\end{aligned}
$$

which is a contradiction.

By Lemma 2.3, we can write $N=N^{+} \cup N^{-}$for all

Define

$$
\left(|\lambda|\|f\|_{\infty}\right)^{\frac{p}{p-q}}+\left(|\mu|\|g\|_{\infty}\right)^{\frac{p}{p-q}} \in\left(0, \Lambda_{1}\right)
$$

$$
\alpha^{+}=\inf _{(u, v) \in N^{+}} I(u, v), \alpha^{-}=\inf _{(u, v) \in N^{-}} I(u, v) .
$$

Lemma 2.4. (i) $\alpha^{+}<0$ for all

$$
\left(|\lambda|\|f\|_{\infty}\right)^{\frac{p}{p-q}}+\left(|\mu|\|g\|_{\infty}\right)^{\frac{p}{p-q}} \in\left(0, \Lambda_{1}\right)
$$

(ii) There exists a positive constant $d_{0}$ depending on $\lambda, \mu, q, N, S, \bar{S}, f, g, \alpha, \beta$ such that $\alpha^{-}>d_{0}$ for all

$$
\left(\mid \lambda\|\| f \|_{\infty}\right)^{\frac{p}{p-q}}+\left(|\mu|\|g\|_{\infty}\right)^{\frac{p}{p-q}} \in\left(0,\left(\frac{q}{p}\right)^{\frac{p}{p-q}} \Lambda_{1}\right) .
$$

Proof. (i) Suppose $(u, v) \in N^{+}$, then we have $I(u, v)$

$$
\begin{aligned}
& =\left(\frac{1}{p}-\frac{1}{q}\right)\|(u, v)\|_{W}^{p}+\left(\frac{1}{q}-\frac{1}{\alpha+\beta}\right) G(u, v) \\
& <\left(\frac{1}{p}-\frac{1}{q}\right)\|(u, v)\|_{W}^{p}+\left(\frac{1}{q}-\frac{1}{\alpha+\beta}\right)\left(\frac{p-q}{\alpha+\beta-q}\right)\|(u, v)\|_{W}^{p} \\
& =-\frac{(\alpha+\beta-p)(p-q)}{(\alpha+\beta) p q}\|(u, v)\|_{W}^{p}<0
\end{aligned}
$$

for $1<q<p<\alpha+\beta$.

Thus we get that $\alpha^{+}<0$.

(ii) Suppose

$$
\left(|\lambda|\|f\|_{\infty}\right)^{\frac{p}{p-q}}+\left(|\mu|\|g\|_{\infty}\right)^{\frac{p}{p-q}} \in\left(0,\left(\frac{q}{p}\right)^{\frac{p}{p-q}} \Lambda_{1}\right),
$$

and $(u, v) \in N^{-}$. Then (2.4) implies that

$$
\|(u, v)\|_{W}>\left[\frac{p-q}{\alpha+\beta-q} S^{\frac{\alpha+\beta}{p}}\right]^{\frac{1}{\alpha+\beta-p}},
$$

and (2.5) implies that

$$
\begin{aligned}
R(u, v) \leq \bar{S}^{-\frac{q}{p}}\|(u, v)\|_{W}^{q} & \\
& \cdot\left[\left(|\lambda|\|f\|_{\infty}\right)^{\frac{p}{p-q}}+\left(|\mu|\|g\|_{\infty}\right)^{\frac{p}{p-q}}\right]^{\frac{p-q}{p}} .
\end{aligned}
$$


From (2.7) and (2.8) it follows that

$$
\begin{aligned}
& I(u, v)=\left(\frac{1}{p}-\frac{1}{\alpha+\beta}\right)\|(u, v)\|_{W}^{p}+\left(\frac{1}{\alpha+\beta}-\frac{1}{q}\right) R(u, v) \\
& \geq \frac{\alpha+\beta-p}{(\alpha+\beta) p}\|(u, v)\|_{W}^{p}-\frac{\alpha+\beta-q}{(\alpha+\beta) q} \bar{S}^{-\frac{q}{p}}\|(u, v)\|_{W}^{q} \\
& {\left[\left(|\lambda|\|f\|_{\infty}\right)^{\frac{p}{p-q}}+\left(\mu\|g\|_{\infty}\right)^{\frac{p}{p-q}}\right]^{\frac{p-q}{p}}=\|(u, v)\|_{W}^{q}} \\
& \left\{\frac{\alpha+\beta-p}{(\alpha+\beta) p}\|(u, v)\|_{W}^{p-q}\right. \\
& \left.-\frac{\alpha+\beta-q}{(\alpha+\beta) q} \bar{S}^{-\frac{q}{p}}\left[\left(\lambda\|f\|_{\infty}\right)^{\frac{p}{p-q}}+\left(\mu\|g\|_{\infty}\right)^{\frac{p}{p-q}}\right]^{\frac{p-q}{p}}\right\} \\
& >\left[\frac{p-q}{\alpha+\beta-q} S^{\frac{\alpha+\beta}{p}}\right]^{\frac{q}{\alpha+\beta-p}} \\
& \left\{\frac{\alpha+\beta-p}{(\alpha+\beta) p}\left[\frac{p-q}{\alpha+\beta-q} S^{\frac{\alpha+\beta}{p}}\right]^{\frac{p-q}{\alpha+\beta-p}}\right. \\
& \left.-\bar{S}^{-\frac{q}{p}} \frac{\alpha+\beta-q}{(\alpha+\beta) q}\left[\left(|\lambda|\|f\|_{\infty}\right)^{\frac{p}{p-q}}+\left(\mu\|g\|_{\infty}\right)^{\frac{p}{p-q}}\right]^{\frac{p-q}{p}}\right\}
\end{aligned}
$$

which shows that

$$
I(u, v)>d_{0}, \forall(u, v) \in N^{-},
$$

since $\left(|\lambda|\|f\|_{\infty}\right)^{\frac{p}{p-q}}+\left(|\mu|\|g\|_{\infty}\right)^{\frac{p}{p-q}} \in\left(0,\left(\frac{q}{p}\right)^{\frac{p}{p-q}} \Lambda_{1}\right)$,

where $d_{0}=d_{0}(\lambda, \mu, q, N, S, \bar{S}, f, g, \alpha, \beta)$ is a positive constant.

For all $(u, v) \in W$ such that $G(u, v)>0$, set

$$
t_{\max }=\left(\frac{(p-q)\|(u, v)\|_{W}^{p}}{(\alpha+\beta-q) G(u, v)}\right)^{\frac{1}{\alpha+\beta-p}}>0 .
$$

Lemma 2.5. Suppose that

$$
\left(|\lambda|\|f\|_{\infty}\right)^{\frac{p}{p-q}}+\left(|\mu|\|g\|_{\infty}\right)^{\frac{p}{p-q}} \in\left(0, \Lambda_{1}\right)
$$

and $(u, v) \in W$ is a function satisfying $G(u, v)>0$.

(i) If $R(u, v) \leq 0$, then there exists a unique $t_{1}>t_{\max }$ such that $\left(t_{1} u, t_{1} v\right) \in N^{-}$and

$I\left(t_{1} u, t_{1} v\right)=\sup _{t \geq 0} I(t u, t v)$.

(ii) If $R(u, v)>0$, then there exist $t_{2}$ and $t_{3}$ such that

$$
0<t_{2}<t_{\max }<t_{3},\left(t_{2} u, t_{2} v\right) \in N^{+} \text {and }\left(t_{3} u, t_{3} v\right) \in N^{-} .
$$

Furthermore, $I\left(t_{2} u, t_{2} v\right)=\inf _{0 \leq t \leq t_{\max }} I(t u, t v)$,

$I\left(t_{3} u, t_{3} v\right)=\sup _{t \geq 0} I(t u, t v)$.

Proof. Fix $(u, v) \in W$ with $G(u, v)>0$. For all $t \geq 0$, let

$$
\Psi(t)=t^{p-q}\|(u, v)\|_{W}^{p}-t^{\alpha+\beta-q} G(u, v),
$$

then it is obvious that $\Psi(0)=0, \Psi(t) \rightarrow-\infty$ as $t \rightarrow+\infty$, $\Psi(t)>0$ as $t>0$ small enough. So we can deduce that $\Psi^{\prime}(t)=0$ at $t=t_{\max }, \Psi^{\prime}(t)>0$ for $t \in\left(0, t_{\max }\right)$, $\Psi^{\prime}(t)<0$ for $t \in\left(t_{\max },+\infty\right)$. Then $\Psi(t)$ that achieves its maximum at $t_{\max }$ is increasing for $t \in\left(0, t_{\max }\right)$ and decreasing for $t \in\left(t_{\max },+\infty\right)$. Moreover,

$$
\begin{aligned}
\Psi & \left(t_{\max }\right) \\
= & \left(\frac{(p-q)\|(u, v)\|_{W}^{p}}{(\alpha+\beta-q) G(u, v)}\right)^{\frac{p-q}{\alpha+\beta-p}}\|(u, v)\|_{W}^{p} \\
& -\left(\frac{(p-q)\|(u, v)\|_{W}^{p}}{(\alpha+\beta-q) G(u, v)}\right)^{\frac{\alpha+\beta-q}{\alpha+\beta-p}} G(u, v) \\
= & {\left.\left[\frac{(p-q)}{(\alpha+\beta-q)}\right)^{\frac{p-q}{\alpha+\beta-p}}-\left(\frac{(p-q)}{(\alpha+\beta-q)}\right)^{\frac{\alpha+\beta-q}{\alpha+\beta-p}}\right] } \\
& \times\left(\frac{\|(u, v)\|_{W}^{\alpha+\beta}}{G(u, v)}\right)^{\frac{p-q}{\alpha+\beta-q}}\|(u, v)\|_{W}^{q} \\
\geq & {\left[\frac{p-q}{\alpha+\beta-q} S \frac{\alpha+\beta}{p}\right]^{\frac{p-q}{\alpha+\beta-p}} \frac{\alpha+\beta-p}{\alpha+\beta-q}\|(u, v)\|_{W}^{q}>0 . }
\end{aligned}
$$

(i) If $R(u, v) \leq 0$, then there exists a unique $t_{1}>t_{\max }>0 \quad$ such that $\Psi\left(t_{1}\right)=R(u, v) \leq 0, \Psi^{\prime}\left(t_{1}\right)<0$. Note that

$$
\begin{aligned}
& \left\langle I^{\prime}\left(t_{1} u, t_{1} v\right),\left(t_{1} u, t_{1} v\right)\right\rangle \\
& =t_{1}^{p}\|(u, v)\|_{W}^{p}-t_{1}^{q} R(u, v)-t_{1}^{\alpha+\beta} G(u, v) \\
& =t_{1}^{q}\left(t_{1}^{p-q}\|(u, v)\|_{W}^{p}-t_{1}^{\alpha+\beta-q} G(u, v)-R(u, v)\right) \\
& =t_{1}^{q}\left(\Psi\left(t_{1}\right)-R(u, v)\right)=0,
\end{aligned}
$$

thus we get $\left(t_{1} u, t_{1} v\right) \in N$.

From

$$
\begin{aligned}
& \Phi^{\prime}\left(t_{1} u, t_{1} v\right),\left(t_{1} u, t_{1} v\right) \\
& =(p-q) t_{1}^{p}\|(u, v)\|_{W}^{p}-(\alpha+\beta-q) t_{1}^{\alpha+\beta} G(u, v) \\
& =t_{1}^{1+q} \Psi^{\prime}\left(t_{1}\right)<0,
\end{aligned}
$$


we have $\left(t_{1} u, t_{1} v\right) \in N^{-}$. For all $t>t_{\max }$, it follows that

$$
\begin{aligned}
& \Phi^{\prime}(t u, t v),(t u, t v) \\
& =(p-q) t^{p}\|(u, v)\|_{W}^{p}-(\alpha+\beta-q) t^{\alpha+\beta} G(u, v) \\
& =t^{1+q} \Psi^{\prime}(t)<0, \\
& \frac{\mathrm{d}^{2}}{\mathrm{~d} t^{2}} I(t u, t v)<0, \\
& \frac{\mathrm{d}}{\mathrm{d} t} I(t u, t v)=t^{q-1}(\Phi(t)-R(u, v))=0 \text { for } t=t_{1} .
\end{aligned}
$$

So we get that $I\left(t_{1} u, t_{1} v\right)=\sup I(t u, t v)$.

(ii) If $R(u, v)>0$, for

$$
\begin{aligned}
&\left(\mid \lambda\|\| f \|_{\infty}\right)^{\frac{p}{p-q}}+\left(|\mu|\|g\|_{\infty}\right)^{\frac{p}{p-q}} \in\left(0, \Lambda_{1}\right), \\
& \Psi(0)=0<R(u, v) \\
& \leq \bar{S}^{-\frac{q}{p}}\left[\left(\lambda\|f\|_{\infty}\right)^{\frac{p}{p-q}}+\left(\mu\|g\|_{\infty}\right)^{\frac{p}{p-q}}\right]^{\frac{p-q}{p}}\|(u, v)\|_{W}^{q} \\
&<\bar{S}^{-\frac{q}{p}} \Lambda_{1}^{\frac{p-q}{p}}\|(u, v)\|_{W}^{q} \\
&=\left[\frac{p-q}{\alpha+\beta-q} S^{\frac{\alpha+\beta}{p}}\right]^{\frac{p-q}{\alpha+\beta-p}} \frac{\alpha+\beta-p}{\alpha+\beta-q}\|(u, v)\|_{W}^{q} \\
& \leq \Psi\left(t_{\max }\right),
\end{aligned}
$$

then there exist $t_{2}$ and $t_{3}$ such that

$0<t_{2}=t_{2}(u, v)<t_{\max }<t_{3}, \Psi\left(t_{2}\right)=R(u, v)=\Psi\left(t_{3}\right)$ and $\Psi^{\prime}\left(t_{2}\right)>0>\Psi^{\prime}\left(t_{3}\right)$. By the similar argument in (i), we get $\left(t_{2} u, t_{2} v\right) \in N^{+},\left(t_{3} u, t_{3} v\right) \in N^{-}$, and

$$
\begin{aligned}
& \frac{\mathrm{d}}{\mathrm{d} t} I(t u, t v)=0 \text { for } t=t_{2} \text { or } t=t_{3} . \\
& \frac{\mathrm{d}^{2}}{\mathrm{~d} t^{2}} I(t u, t v)>0 \text { for } t \in\left(0, t_{\max }\right) \\
& \frac{\mathrm{d}^{2}}{\mathrm{~d} t^{2}} I(t u, t v)<0 \text { for } t=\left(t_{\max },+\infty\right) .
\end{aligned}
$$

Then it follows that

$$
\begin{aligned}
& I\left(t_{2} u, t_{2} v\right)=\inf _{0 \leq t \leq t_{\max }} I(t u, t v), \\
& I\left(t_{3} u, t_{3} v\right)=\sup _{t \geq 0} I(t u, t v) .
\end{aligned}
$$

The proof of this Lemma is completed.

For each $(u, v) \in W$ with $R(u, v)>0$, we write

$$
\bar{t}_{\max }=\left(\frac{(\alpha+\beta-q) R(u, v)}{(\alpha+\beta-p)\|(u, v)\|_{W}^{p}}\right)^{\frac{1}{p-q}}>0 .
$$

Lemma 2.6. Suppose that

$$
\left(|\lambda|\|f\|_{\infty}\right)^{\frac{p}{p-q}}+\left(|\mu|\|g\|_{\infty}\right)^{\frac{p}{p-q}} \in\left(0,\left(\frac{q}{p}\right)^{\frac{p}{p-q}} \Lambda_{1}\right)
$$

and $(u, v) \in W$ is a function satisfying $R(u, v)>0$.

(i) If $G(u, v) \leq 0$, then there exists a unique $t_{1}>\bar{t}_{\max }$ such that $\left(t_{1} u, t_{1} v\right) \in N^{-}$and

$$
I\left(t_{1} u, t_{1} v\right)=\sup _{t \geq \bar{t}_{\max }} I(t u, t v) .
$$

(ii) If $G(u, v)>0$, then there exist $t_{2}$ and $t_{3}$ such that $0<t_{2}<\bar{t}_{\max }<t_{3},\left(t_{2} u, t_{2} v\right) \in N^{+}$and $\left(t_{3} u, t_{3} v\right) \in N^{-}$. Furthermore,

$$
\begin{aligned}
& I\left(t_{2} u, t_{2} v\right)=\inf _{0 \leq t \leq t_{\max }} I(t u, t v) \\
& I\left(t_{3} u, t_{3} v\right)=\sup _{t \geq 0} I(t u, t v)
\end{aligned}
$$

Proof. Fix $(u, v) \in W$ with $R(u, v)>0$. For all $t>0$, let

$$
\bar{\Psi}(t)=t^{p-\alpha-\beta}\|(u, v)\|_{W}^{p}-t^{q-\alpha-\beta} R(u, v)
$$

then it is obvious that $\bar{\Psi}(t) \rightarrow-\infty$ as $t \rightarrow 0+$. So we can deduce that $\bar{\Psi}^{\prime}(t)=0$ at $t=\bar{t}_{\max }$,

$\bar{\Psi}^{\prime}(t)>0$ for $t \in\left(0, \bar{t}_{\max }\right), \quad \bar{\Psi}^{\prime}(t)<0$ for $t \in\left(\bar{t}_{\max },+\infty\right)$. Then $\bar{\Psi}(t)$ that achieves its maximum at $\bar{t}_{\max }$ is increasing for $t \in\left(0, \bar{t}_{\max }\right)$ and decreasing for $t \in\left(\bar{t}_{\max },+\infty\right)$. Using the similar argument in Lemma 2.5, we can obtain the result of Lemma 2.6.

\section{Proof of Theorem 1.1}

Lemma 3.1. Suppose that

$$
\left(|\lambda|\|f\|_{\infty}\right)^{\frac{p}{p-q}}+\left(|\mu|\|g\|_{\infty}\right)^{\frac{p}{p-q}} \in\left(0, \Lambda_{1}\right),
$$

then the functional I has a minimizer $(u, v) \in N^{+}$and it satisfies

(i) $I(u, v)=\alpha^{+}$.

(ii) $(u, v)$ is a nontrivial solution of (1.1).

Proof. Let $\left\{\left(u_{n}, v_{n}\right)\right\} \subset N^{+}$be a minimizing sequence such that

$$
I\left(u_{n}, v_{n}\right)=\alpha^{+}+o_{n}(1), I^{\prime}\left(u_{n}, v_{n}\right)=o_{n}(1) .
$$

Since $I$ is coercive on $N$, we get that $\left\{\left(u_{n}, v_{n}\right)\right\}$ is bounded on $W$. Passing to a subsequence (still denoted by $\left.\left\{\left(u_{n}, v_{n}\right)\right\}\right)$, there exists $(u, v)$ such that

$$
\begin{aligned}
& \left(u_{n}, v_{n}\right) \rightarrow(u, v) \text { weakly in } W, \\
& u_{n} \rightarrow u, v_{n} \rightarrow v \text { a.e. in } \Omega,
\end{aligned}
$$

$u_{n} \rightarrow u, v_{n} \rightarrow v$ strongly in $L^{q}(\partial \Omega)$ and in $L^{\alpha+\beta}(\Omega)$. This implies

$$
R\left(u_{n}, v_{n}\right) \rightarrow R(u, v), G\left(u_{n}, v_{n}\right) \rightarrow G(u, v) .
$$


Since $\left\{\left(u_{n}, v_{n}\right)\right\} \subset N^{+}$, we get

$$
I\left(u_{n}, v_{n}\right)=\frac{\alpha+\beta-p}{p(\alpha+\beta)}\|(u, v)\|_{W}^{q}-\frac{\alpha+\beta-q}{q(\alpha+\beta)} R\left(u_{n}, v_{n}\right)
$$

By Lemma 2.4 (i) we get $I\left(u_{n}, v_{n}\right) \rightarrow \alpha^{+}<0$ and then $R(u, v)>0$. Now we prove that $\left(u_{n}, v_{n}\right) \rightarrow(u, v)$ strongly in $W$. Suppose otherwise, then either

$$
\|u\|_{1, p}<\liminf _{n \rightarrow \infty}\left\|u_{n}\right\|_{1, p} \text { or }\|v\|_{1, p}<\liminf _{n \rightarrow \infty}\left\|v_{n}\right\|_{1, p} .
$$

Fix $(u, v) \in W$ with $R(u, v)>0$. Let $E(t)=\bar{\Psi}(t)-G(u, v)$, where $\bar{\Psi}(t)$ is as in (2.10).

Clearly, $E(t) \rightarrow-\infty$ as $t \rightarrow 0+$, and $E(t) \rightarrow-G(u, v)$ as $t \rightarrow \infty$. Since $E^{\prime}(t)=\bar{\Psi}^{\prime}(t)$, by an argument similar to the one in the proof of Lemma 2.6, we have that the function $E(t)$ achieves its maximum at $\bar{t}_{\max }$, is increasing for $t \in\left(0, \bar{t}_{\max }\right)$ and decreasing for $t \in\left(\bar{t}_{\max },+\infty\right)$, where $\bar{t}_{\max }$ is as in (2.9). Since $R(u, v)>0$, by Lemma 2.6, there is unique $0<t_{0}<\bar{t}_{\max }$ such that

$$
\left(t_{0} u, t_{0} v\right) \in N^{+}, I\left(t_{0} u, t_{0} v\right)=\inf _{0 \leq t \leq t_{\max }} I(t u, t v),
$$

Then

$$
\begin{aligned}
& E\left(t_{0}\right)=\bar{\Psi}\left(t_{0}\right)-G(u, v) \\
& =t_{0}^{-\alpha-\beta}\left[\left\|\left(t_{0} u, t_{0} v\right)\right\|_{W}^{p}-R\left(t_{0} u, t_{0} v\right)-G\left(t_{0} u, t_{0} v\right)\right] \\
& =0 .
\end{aligned}
$$

By (3.3) and (3.4), we obtain $E\left(t_{0}\right)>0$ for $n$ sufficiently large for the sequence $\left\{\left(u_{n}, v_{n}\right)\right\}$. Since $\left(u_{n}, v_{n}\right) \in N^{+}$, we have $\bar{t}_{\max }\left(u_{n}, v_{n}\right)>1$. Moreover,

$$
E(1)=\left\|\left(u_{n}, v_{n}\right)\right\|_{W}^{p}-R\left(u_{n}, v_{n}\right)-G\left(u_{n}, v_{n}\right)=0,
$$

and $E(t)$ is increasing for $t \in\left(0, \bar{t}_{\max }\left(u_{n}, v_{n}\right)\right)$. This implies $E(t)<0$ for all $t \in(0,1]$ and $n$ sufficiently large. We obtain $1<t_{0}<\bar{t}_{\max }(u, v)$. But $\left(t_{0} u, t_{0} v\right) \in N^{+}$ and $I\left(t_{0} u, t_{0} v\right)=\inf _{0 \leq t \leq \bar{t}_{\max }} I(t u, t v)$, this implies

$$
I\left(t_{0} u, t_{0} v\right)<I(u, v)<\lim _{n \rightarrow \infty} I\left(u_{n}, v_{n}\right),
$$

which is a contradiction. Hence $\left(u_{n}, v_{n}\right) \rightarrow(u, v)$ strongly in $W$. This implies $I\left(u_{n}, v_{n}\right) \rightarrow I(u, v)=\alpha^{+}$as $n \rightarrow \infty$. Thus $(u, v)$ is a minimizer for $I$ on $N^{+}$. Since $I(u, v)=I(|u|,|v|)$ and $(|u|,|v|) \in N^{+}$, by Lemma 2.2 we may assume that $(u, v)$ is a nontrivial nonnegative solution of Equation (1.1).

Next we prove $u \neq 0, v \neq 0$. Arguing by contradiction, without loss of generality, we may assume that $v \equiv 0$. Then as $u$ is a nonzero solution of

$$
\left\{\begin{array}{l}
-\Delta_{p} u+m(x)|u|^{p-2} u=0, x \in \Omega, \\
|\nabla u|^{p-2} \frac{\partial u}{\partial \mathbf{n}}=\lambda f(x)|u|^{q-2} u, x \in \partial \Omega
\end{array}\right.
$$

we have

$$
\|(u, 0)\|_{W}^{p}=\lambda \int_{\partial \Omega} f(x)|u|^{q} \mathrm{~d} s \geq 0 .
$$

Choose $z \in W^{1, p}(\Omega) \backslash\{0\}$ such that

$$
\|(0, z)\|_{W}^{p}=\lambda \int_{\partial \Omega} g(x)|z|^{q} \mathrm{~d} s>0,
$$

then

$$
\lambda \int_{\partial \Omega} f(x)|u|^{q} \mathrm{~d} s+\mu \int_{\partial \Omega} g(x)|z|^{q} \mathrm{~d} s>0 .
$$

By Lemma 2.6, there is a unique $0<\bar{t}<\bar{t}_{\max }$ such that $(\bar{t} u, \overline{t z}) \in N^{+}$. Moreover, from (3.6) and (3.7), it follows that

$$
\begin{aligned}
\bar{t}_{\max } & =\left(\frac{(\alpha+\beta-q) R(u, z)}{(\alpha+\beta-p)\|(u, z)\|_{W}^{p}}\right)^{\frac{1}{p-q}} \\
& =\left(\frac{\alpha+\beta-q}{\alpha+\beta-p}\right)^{\frac{1}{p-q}}>1,
\end{aligned}
$$

and

$$
I(\overline{t u}, \overline{t z})=\inf _{0 \leq t \leq \bar{t}_{\max }} I(t u, t z) .
$$

This implies

$$
I(\bar{t} u, \bar{t} v) \leq I(u, v)<I(u, 0),
$$

which contradict with that $(u, 0)$ is the minimizer and hence $u \neq 0, v \neq 0$. So $(u, v)$ is a nontrivial nonnegative solution of Equation (1.1).

Lemma 3.2. Suppose that

$$
\left(|\lambda|\|f\|_{\infty}\right)^{\frac{p}{p-q}}+\left(|\mu|\|g\|_{\infty}\right)^{\frac{p}{p-q}} \in\left(0,\left(\frac{q}{p}\right)^{\frac{p}{p-q}} \Lambda_{1}\right) .
$$

Then the functional I has a minimizer $(U, V) \in N^{-}$ and it satisfies

(i) $I(U, V)=\alpha^{-}$.

(ii) $(U, V)$ is a nontrivial solution of (1.1).

Proof. Let $\left\{\left(u_{n}, v_{n}\right)\right\} \subset N^{-}$be a minimizing sequence such that

$$
I\left(u_{n}, v_{n}\right)=\alpha^{-}+o_{n}(1), I^{\prime}\left(u_{n}, v_{n}\right)=o_{n}(1) .
$$

Since $I$ is coercive on $N$, we get that $\left\{\left(u_{n}, v_{n}\right)\right\}$ is bounded on $W$. Passing to a subsequence (still denoted by $\left.\left\{\left(u_{n}, v_{n}\right)\right\}\right)$, there exists $(U, V)$ such that

$$
\begin{gathered}
\left(u_{n}, v_{n}\right) \rightarrow(U, V) \text { weakly in } W, \\
u_{n} \rightarrow U, v_{n} \rightarrow V \text { a.e. in } \Omega,
\end{gathered}
$$


$u_{n} \rightarrow U, v_{n} \rightarrow V$ strongly in $L^{q}(\partial \Omega)$ and in $L^{\alpha+\beta}(\Omega)$.

This implies

$$
R\left(u_{n}, v_{n}\right) \rightarrow R(U, V), G\left(u_{n}, v_{n}\right) \rightarrow G(U, V) .
$$

Moreover, by (2.3) we obtain

$$
G\left(u_{n}, v_{n}\right)>\frac{p-q}{\alpha+\beta-q}\left\|\left(u_{n}, v_{n}\right)\right\|_{W}^{p},
$$

then $G(U, V) \geq C>0$. Now we prove that $\left(u_{n}, v_{n}\right) \rightarrow(U, V)$ strongly in $W$. Suppose otherwise, then either

$$
\begin{aligned}
& \|U\|_{1, p}<\liminf _{n \rightarrow \infty}\left\|u_{n}\right\|_{1, p} \\
& \text { or }\|V\|_{1, p}<\liminf _{n \rightarrow \infty}\left\|v_{n}\right\|_{1, p} .
\end{aligned}
$$

By Lemma 2.6, there is unique $\mathrm{t}_{\mathrm{o}}$ such that $\left(t_{0} U, t_{0} V\right) \in N^{-}$, Since $\left(u_{n}, v_{n}\right) \in N^{-}, I\left(u_{n}, v_{n}\right) \geq I\left(t u_{n}, t v_{n}\right)$ for all $t \geq 0$, we have

$$
I\left(t_{0} U, t_{0} V\right)<\lim _{n \rightarrow \infty} I\left(t_{0} u_{n}, t_{0} v_{n}\right) \leq \lim _{n \rightarrow \infty} I\left(u_{n}, v_{n}\right)=\alpha^{-},
$$

and this is a contradiction. Hence $\left(u_{n}, v_{n}\right) \rightarrow(U, V)$ strongly in $W$. This implies $I\left(u_{n}, v_{n}\right) \rightarrow I(U, V)=\alpha^{-}$ as $n \rightarrow \infty$. Thus $(U, V)$ is a minimizer for $I$ on $N^{-}$. Since $I(U, V)=I(|U|,|V|)$ and $(|U|,|V|) \in N^{-}$, by Lemma 2.4 and the similar argument as that in Lemma 3.1 we can get $(U, V)$ is also a nontrivial nonnegative solution of Equation (1.1).

Proof of Theorem 1.1. From Lemma 3.1 and Lemma 3.2, we obtain that Equation (1.1) has two nontrivial nonnegative solutions $(u, v)$ and $(U, V)$ satisfy $(u, v) \in N^{+}$and $(U, V) \in N^{-}$. It remains to show that the solutions found in Lemma 3.1 and Lemma 3.2 are distinct. Since $N^{+} \cap N^{-}=\varnothing$, this implies that $(u, v)$ and $(U, V)$ are distinct. This concludes the proof.

\section{Acknowledgements}

The author is indebted to the referees for carefully reading this paper and making valuable comments and suggestions.

\section{REFERENCES}

[1] J. Garcia-Azorero, I. Peral and J. D. Rossi, "A ConvexConcave Problem with a Nonlinear Boundary Condition," Journal of Differential Equations, Vol. 198, No. 1, 2004, pp. 91-128. doi:10.1016/S0022-0396(03)00068-8.

[2] T.-F. Wu, "A Semilinear Elliptic Problem Involving Nonlinear Boundary Condition and Sign-Changing Potential," Electronic Journal of Differential Equations, Vol. 2006, No. 131, 2006, pp. 1-15.

[3] T.-F. Wu, "Multiplicity of Positive Solution of p-Laplacian Problems with Sign-Changing Weight Functions," International Journal of Mathematical Analysis, Vol. 1, No. 9-12, 2007, pp. 557-563.

[4] T.-F. Wu, "Multiple Positive Solutions for Semilinear Elliptic Systems with Nonlinear Boundary Condition," Applied Mathematics and Computation, Vol. 189, No. 2, 2007, pp. 1712-1722. doi:10.1080/028418501127346846.

[5] T.-S. Hsu, "Multiple Positive Solutions for a Critical Quasilinear Elliptic System with Oncave-Convex Nonlinearitiesc," Nonlinear Analysis, Vol. 71, No. 7-8, 2009, pp. 2688-2698. doi:10.1016/ j.na.2009.01.110.

[6] T.-F. Wu, "On Semilinear Elliptic Equations Involving Concave-Convex Nonlinearities and Sign-Changing Weight Function," Article Journal of Mathematical Analysis and Applications, Vol. 318, No. 1, 2006, pp. 253-270. doi:10.1016/j.jmaa. 2005.05.057.

[7] T.-S. Hsu and H.-L. Lin, "Multiple Positive Solutions for Singular Elliptic Equations with Concave-Convex Nonlinearities and Sign-Changing Weights," Boundary Value Problems, Vol. 2009, 2009, Article ID: 584203.

[8] L. Wang, Q. Wei and D. Kang, "Existence and Multiplicity of Positive Solutions to Elliptic Systems Involving Critical Exponents," Journal of Mathematical Analysis and Applications, Vol. 383, No. 2, 2011, pp. 541-552. doi:10.1016/j.jmaa. 2011.05.053.

[9] K. Brown and Y. Zhang, "The Nehari Manifold for a Semilinear Elliptic Equation with a Sign-Changing Weight Function," Journal of Differential Equations, Vol. 193, No. 2, 2003, pp. 481-499. doi:10.1016/S0022-0396(03)00121-9.

[10] G. Tarantello, "On Nonhomogeneous Elliptic Equations Involving Critical Sobolev Exponent," Annales De L Institut Henri Poincare-Analyse Non Lineaire, Vol. 9, No. 3, 1992, pp. 281-304. 